Cultures \& Conflits

46 | été 2002

De Tampere à Séville : bilan de la sécurité européenne $(2 / 2)$

\title{
Le pacte de stabilité pour l'Europe du sud-est
}

The Southeast Europe Stability Pact

\section{Anastassia Tsoukala}

\section{(2) OpenEdition}

Journals

Édition électronique

URL : http://journals.openedition.org/conflits/807

DOI : $10.4000 /$ conflits.807

ISSN : $1777-5345$

Éditeur :

CCLS - Centre d'études sur les conflits lilberté et sécurité, L'Harmattan

Édition imprimée

Date de publication : 1 juin 2002

Pagination : 117-125

ISBN : 2-7475-3030-2

ISSN : 1157-996X

Référence électronique

Anastassia Tsoukala, «Le pacte de stabilité pour l'Europe du sud-est », Cultures \& Conflits [En ligne], 46 | été 2002, mis en ligne le, consulté le 30 mars 2021. URL : http://journals.openedition.org/conflits/ 807 ; DOI : https://doi.org/10.4000/conflits.807

Ce document a été généré automatiquement le 30 mars 2021.

Creative Commons License 


\title{
Le pacte de stabilité pour l'Europe du sud-est
}

\author{
The Southeast Europe Stability Pact
}

Anastassia Tsoukala

Bien que la nécessité de renforcer la coopération régionale dans les Balkans ait été déjà manifeste à la fin des années 1980, comme nous l'indique l'organisation d'une réunion des ministres des Affaires étrangères de six pays balkaniques, qui a eu lieu en 1988, à Belgrade, ce n'est qu'après la fin de la bipolarité que nous assistons au lancement d'une série d'initiatives visant à promouvoir la stabilité, la sécurité et la prospérité de la région ${ }^{1}$.

Ainsi, dans les années 1990, nombre de pays balkaniques adhèrent à des structures de coopération régionale plus amples, telles que la Central European Initiative (CEI) et la Black Sea Economic Co-operation(BSEC). Relancée après les Accords de Dayton de 1995, la coopération régionale fut promue par des structures nouvelles, telles que le Royaumont Process de 1995 (au sein duquel on examinait la possibilité de faire appliquer aux Balkans les idées promues par le Pacte de Stabilité en Europe qui se développait alors sous l'égide de l'OSCE), et la Southeast European Co-operation Initiative (SECI), qui visait à encourager la coopération régionale afin de favoriser l'intégration des pays balkaniques dans le cadre européen.

La structure la plus récente en la matière est le Pacte de Stabilité pour l'Europe du Sud-Est, signé à Cologne le 10 juin 1999, qui vise à fournir à tous les pays intéressés le cadre pour atteindre les objectifs communs dans les domaines de la démocratisation et des droits de l'homme, avec une attention particulière aux droits des minorités (table de travail I), du développement et de la restructuration économique (table de travail II) et de la sécurité intérieure et extérieure (table de travail III)2.

Bien qu'il soit encore précoce d'évaluer les résultats du Pacte, il est clair que celui-ci ne vise pas à créer un nouveau réseau de coopération, mais à restructurer et promouvoir les réseaux tissés par les accords régionaux préexistants, en coordonnant leur action avec celle de nouveaux acteurs et en les transformant en véritables piliers de sa propre structure. Notre article se limitant à mettre en lumière les mécanismes récents mis en 
place en matière de contrôle des frontières et de lutte contre la criminalité organisée dans les Balkans, nous nous focaliserons sur le volet sécuritaire du Pacte, prévu par la troisième table de travail, et notamment la première sous-table de celle-ci, qui s'occupe des questions relatives à la sécurité intérieure des pays intéressés.

Nous devons pourtant souligner d'emblée que, loin d'être uniques, les projets inclus dans le Pacte de stabilité s'intègrent dans une foule de projets de renforcement de la coopération régionale, multilatéraux ou bilatéraux, dont un des points communs consiste à lier le développement économique et la démocratisation des pays balkaniques au rétablissement de la loi et de l'ordre dans la région, ce qui implique notamment la lutte contre la criminalité organisée, la corruption et l'immigration clandestine. Nous allons, donc, dans un premier temps, examiner l'approche multilatérale, en nous focalisant sur le Pacte de stabilité et sur deux autres structures liées à celui-ci, la SECI et l'accord d'Adriatique. Ensuite, nous examinerons l'approche bilatérale, en nous focalisant sur les actions entreprises en la matière par l'Italie dans les Balkans et surtout en Albanie.

L'approche multilatérale

Le Pacte de stabilité : la sous-table sur la justice et les affaires intérieures

Le succès du processus de stabilisation régionale et des réformes démocratiques et économiques qu'implique le Pacte de stabilité étant indissociable du rétablissement de la loi et de l'ordre dans la région, la sous-table sur la justice et les affaires intérieures s'est fixée comme objectifs prioritaires les domaines suivants :

- la lutte contre la corruption, la criminalité organisée, le terrorisme et toute forme d'activité illégale : elle passe par la mise en place d'une stratégie globale, appuyée sur des mesures d'assistance de nature légale, institutionnelle et technique. A cette fin, on cherche à effectuer des réformes pénales et à harmoniser les systèmes judiciaires et les droits nationaux entre eux et avec certains textes de loi internationaux, et on renforce la coopération entre les polices régionales et entre celles-ci et celles des pays occidentaux en encourageant l'échange de renseignements, en envisageant la mise en place d'un système d'information analogue au SIS, en incitant les pays concernés à s'introduire dans le cadre d'activité de l'Europol et en mettant l'accent sur la formation des polices locales ;

- asile et migration : la gestion de ces questions passe par l'amélioration des procédures de contrôles frontaliers, par la professionnalisation des agences chargées de ces contrôles et par l'instauration d'un dialogue sur les politiques à adopter en matière de contrôle des frontières ; par ailleurs, l'attention est aussi attirée sur les modes de mise en œuvre des rapatriements en cas d'exode des populations et sur la formation de cadres préparés à gérer nombre de nouveaux phénomènes issus des conflits récents (trafics d'immigrés, prostitution de femmes et de mineurs, mendicité...) ;

- la formation de la police : la réussite de la lutte contre la corruption et la criminalité organisée ne pouvant s'appuyer exclusivement sur les forces extérieures, la formation des polices locales fut vite érigée en point central de toutes les politiques à suivre. Cette formation aurait une triple mission: améliorer la lutte contre des phénomènes criminels graves, constituer une voie de démocratisation des institutions et d'établissement de l'état de droit et affaiblir la position dominante qu'occupent jusqu'à 
présent les forces armées dans la majorité de ces pays en équilibrant mieux le rapport de forces entre celles-ci et les forces de police.

Il est donc prévu, dans un premier temps, la constitution du Southeast European Staff College, qui sera chargé de la formation d'un corps de police spécialisée. La création de cette institution s'inscrira dans une longue série d'initiatives plus ou moins semblables entamées déjà dans les Balkans, notamment dans le cadre de la lutte contre la criminalité organisée, sans qu'elles soient pour autant couronnées de succès. Nous citons à titre indicatif : la formation des polices locales par des agents de la BKA; la présence de l'International Police Task Force des Nations-Unies et de la Multinational Specialist Unit conduite par l'Italie; le programme de Customs and Fiscal Assistance Office sponsorisé par l'UE; la formation des polices locales par des policiers britanniques, l'envoi d'experts américains chargés d'encadrer la constitution des polices bosniaque et kosovare, etc.

Force est pourtant de constater que même dans le cadre du Pacte ces initiatives ne sont pas encore suffisamment coordonnées. Par ailleurs, bien que les phénomènes de corruption et de criminalité organisée aient été identifiés, même au sommet de Sarajevo, comme obstacle majeur aux réformes politiques et économiques nécessaires à la stabilisation de la région, les initiatives lancées en la matière restent encore faibles, sous-payées et souvent se superposent. Il y a des problèmes de coopération entre forces de l'ordre et magistrats: en Bosnie-Herzégovine par exemple, les affaires portées devant les autorités d'une entité ne sont guère communiquées aux autorités de l'autre. Un autre problème est lié au fait que nombre de chefs d'associations criminelles de type mafieux jouissent de la protection des anciens services de renseignements et de certains politiciens nationalistes.

La Southeast European Co-operation Initiative (SECI)

Les mêmes remarques pourraient aussi s'appliquer dans le cas de la SECI, initiative lancée en décembre 1996 par les Etats-Unis afin de renforcer la coopération régionale dans les domaines économique et environnemental et de faciliter l'intégration des pays balkaniques dans les structures européennes.

Ainsi, si nous examinons le domaine de la facilitation du passage des frontières (dont le pays hôte est la Grèce) nous constatons qu'en matière de restructuration des services frontaliers, la SECI a mis en place un groupe de douaniers et des membres de polices des frontières chargé de la formation des services équivalents des pays intéressés (le premier pays à demander cette aide ayant été la Roumanie en 1998). On envisage en outre la mise en œuvre de réformes afin de mieux organiser les services douaniers et policiers et on propose une hausse des salaires des agents chargés de ces tâches, comme le moyen le plus efficace pour lutter contre la corruption.

Il semble pourtant que les tentatives de formation des forces de l'ordre locales n'aient pas connu beaucoup de succès, pour les raisons déjà évoquées. Par ailleurs, la participation des pays membres de l'UE (à l'exception de la Grèce) se fait de manière réticente, les gouvernements concernés ne souhaitant pas leur pleine intégration dans une structure lancée exclusivement par les Etats-Unis et préférant élaborer le projet de la création d'une Académie de police, chargée de la formation des polices locales.

En matière de lutte contre la criminalité transfrontière et la corruption, le Mémorandum of Understanding (rédigé lors de la réunion de Bucarest, en juin 1998) met l'accent sur la collecte, l'analyse et l'échange de renseignements. Le gouvernement roumain a proposé la création d'un Centre à Bucarest, qui regrouperait des officiers de 
liaison de tous les pays membres et, grâce à un système informatisé de collecte de renseignements, coordonnerait les activités de toutes les agences de contrôle des frontières et l'INTERPOL, vivement intéressée par ce projet, a proposé de coopérer avec la SECI afin de mener à bien cette initiative.

En mai 1999, a été signé à Bucarest l'accord de coopération en matière de prévention et de lutte contre la criminalité transfrontière, qui prévoit (art. 13) la création du Centre Régional SECI pour la lutte contre la criminalité transfrontière (communément appelé Crime Centre). Suite à l'entrée en vigueur de l'accord le 1er février 2000, le Crime Centre devient progressivement opérationnel, avec des sections chargées de la lutte contre le trafic de stupéfiants, la traite des êtres humains, les fraudes douanières, les véhicules volés, etc. Par ailleurs, il est envisagé que le Centre élargisse ses activités afin de pouvoir renforcer la coopération judiciaire entre les pays membres.

L'accord de l'Adriatique

Dans les conclusions du sommet de Tampere (art. 24), on prévoyait la conclusion d'un mémorandum d'accord qui, inscrit dans le contexte du Pacte de stabilité, viserait à renforcer la coopération entre la Grèce et l'Italie dans l'Adriatique et la mer Ionienne pour lutter contre la criminalité organisée, les activités des passeurs et la traite des êtres humains.

La mise en application de ce mémorandum a impliqué, dans un premier temps, la rencontre des chefs des principaux corps de police italiens, grecs et albanais et, dans un deuxième temps, l'organisation de la Conférence d'Adriatique (19-21 mai 2000) sur le renforcement de la coopération en mer Adriatique en matière de lutte contre le crime organisé, la contrebande et l'immigration clandestine.

L'approche bilatérale

La plupart des initiatives multilatérales n'ayant pas connu jusqu'à présent de succès, les pays membres de l'UE concernés tendent à opter plutôt pour l'approche bilatérale. Ainsi, l'Italie se focalise-t-elle sur la formation des polices balkaniques (albanaises, bulgares, macédoniennes, roumaines, monténégrines) dans des programmes d'action bilatéraux ou financés par l'UE. Il semble, d'ailleurs, que la coopération bilatérale porte davantage de fruits. En Albanie, par exemple, cette coopération, qui s'appuie sur de nombreux accords bilatéraux, implique les trois principaux corps de police et vise notamment à améliorer la formation des agences de sécurité albanaise, tout en revêtant parfois la forme d'une coopération opérationnelle.

A titre indicatif, la Garde des Finances, agissant sous l'égide de l'UE et de l'UEO, a actuellement 132 agents et 12 navires occupés dans 5 missions différentes en Albanie :

- la mission bilatérale relative aux affaires intérieures, visant à l'amélioration de la structure des forces de police albanaises et à la coordination des opérations ;

- la mission bilatérale relative aux questions financières, qui se charge de la formation des douaniers albanais ;

- CAM (Customs Assistance Mission) et CAM-SEA (sous l'égide de la Commission), qui vise à réorganiser les douanes albanaises en proposant des réformes législatives et de l'assistance directe et en formant le personnel local dans les procédures de contrôle de la circulation des personnes et des biens ; 
- MAPE (Multinational Advisory Police Element), qui vise à contribuer à la réorganisation du ministère de l'Intérieur grâce à la restructuration de sa division de sécurité nationale.

Cette étroite coopération n'a pas pourtant affaibli le contrôle des frontières maritimes italiennes, notamment au canal d'Otrante qui, depuis la deuxième moitié des années 1990, s'est avéré particulièrement perméable face aux flux migratoires en provenance de l'Albanie. Due tant aux crises politiques albanaises qu'au conflit à Kosovo, la hausse constante du nombre d'immigrés clandestins tentant quotidiennement d'entrer en territoire italien, en traversant de nuit le canal d'Otrante, a fini par rendre pratiquement inefficace le puissant dispositif de contrôle mis en place par les forces de police. Mais, contrairement à ce qui s'est produit en 1993, par rapport au contrôle de la frontière italo-slovène (qui fut confié pendant près de deux ans aux Forces armées), le gouvernement n'a pas voulu jusqu'à présent demander le concours des Forces Armées, en les engageant dans une opération précise.

Cela ne signifie pas pour autant que la Marine ne prête pas, de facto, son concours aux forces de police. En effet, lors de ses patrouilles habituelles de contrôle des eaux territoriales italiennes, la Marine peut toujours contribuer, le cas échéant, à la lutte contre l'immigration clandestine, sans que cela constitue une de ses tâches principales. Il faut préciser qu'en raison de la lutte contre l'immigration clandestine, le commandant d'un bâtiment de la Marine peut exercer les fonctions de police judiciaire. La hausse récente du trafic d'immigrés dans le canal d'Otrante ayant, pourtant, obligé la Marine à intervenir de manière beaucoup plus régulière, celle-ci assure à présent de nombreuses patrouilles nocturnes.

Cette intervention de la Marine va de pair avec une puissante mobilisation des forces de police et notamment de la Garde des Finances, qui fournit la quasi totalité des effectifs en la matière. Cette forte implication de la Garde des Finances à la lutte contre l'immigration clandestine est due au fait que celle-ci est considérée comme menaçante à la sécurité socio-économique, à la sécurité militaire (trafic d'armes) et à la sécurité maritime du pays. Par ailleurs, la qualité de son équipement (elle dispose d'environ 500 navires de toute taille, armés et dotés de systèmes de navigation satellitaire, ainsi que de nombreux avions et hélicoptères) lui permet d'opérer de manière indépendante, même sur le plan informationnel, sans que cela exclue pour autant l'exploitation de renseignements fournis, le cas échéant, par la Marine.

La mobilisation de ces effectifs policiers et militaires n'arrivant pourtant guère à assurer une maitrise satisfaisante des flux migratoires, les autorités italiennes ont cherché à éliminer une des causes de cette inefficacité, à savoir l'absence de tout contrôle de l'immigration clandestine de la part des autorités albanaises, laquelle, allant de pair avec l'impossibilité pour les forces de police italiennes d'intervenir dans les eaux territoriales albanaises, rendait le contrôle du canal d'Otrante quasi aléatoire. Ce problème a été récemment contourné suite à la conclusion d'un accord bilatéral avec l'Albanie. En vertu de celui-ci, les forces de police italiennes ont le droit de patrouiller dans les eaux territoriales albanaises et procéder à des arrestations, à des fouilles, à des saisies et à tout autre acte de police nécessaire, pourvu qu'il y ait à bord de leurs navires un agent de la police albanaise.

Cette forme de coopération extrême nous montre en même temps les limites de la coopération bilatérale dans les Balkans. En effet, nous pouvons nous interroger non 
seulement sur l'efficacité réelle de celle-ci, mais aussi sur les logiques qui la soustendent. Bien que l'étendue de notre intervention ne nous permette pas de développer ces questions, nous nous contenterons de souligner que le renforcement de la coopération policière entre l'Italie et l'Albanie a, certes, porté des fruits mais, en même temps, a obligé les contrebandiers, par exemple, de modifier leurs itinéraires, en choisissant comme lieu de départ de leurs trafics le territoire monténégrin plutôt qu'albanais. Ce changement qui oblige, actuellement, les principaux corps de police italiens à renforcer leur coopération avec les instances policières du Monténégro rend évident le problème des limites quantitatives et qualitatives de cette coopération policière. Jusqu'où devra-t-elle et/ou pourra-t-elle aller? Quelle sera son efficacité réelle si le Monténégro, contrairement à ce que nous avons observé en Albanie, refuse d'accorder aux policiers italiens des pouvoirs opérationnels susceptibles de mettre en cause sa souveraineté?

Mais, dans ce cas, nous pouvons aussi nous demander si, étant donné la situation actuelle dans les Balkans, le Monténégro peut effectivement résister aux pressions italiennes, et par voie d'extension européennes, d'autant plus que celles-ci vont de plus en plus de pair avec des mesures de soutien financier et politique. $Y$ aurait-il alors lieu de croire que le renforcement actuel de la coopération policière dans les Balkans sert en même temps, indirectement, des intérêts autres, dans la mesure où il permettrait la transformation de pays balkaniques en véritables protectorats, étendant de la sorte les zones d'influence de certains pays européens?

\section{NOTES}

1. . Papier présenté à la table ronde organisée par le CERI sur le thème Amsterdam et Tampere, quelles inflexions ?, Paris, 9-10 juin 2000, et publié dans Bürgerrechte und Polizei, 2000, 3, pp. 63-69.

2. . Au Pacte de stabilité ont adhéré les quinze pays membres de l'Union européenne, l'Albanie, la Bosnie-Herzégovine, la Bulgarie, la Croatie, la Hongrie, la Roumanie, la Fédération russe, la Slovénie, la République de Macédoine, la Turquie, les Etats-Unis, la Commission européenne, le Conseil de l'Europe et le Président en charge de l'OSCE. Le Pacte bénéficie aussi du soutien du Canada, du Japon, de l'ONU, de l'UNHCR, de l'OTAN, de l'OCDE, du FMI, de la Banque mondiale, de la Banque européenne pour les investissements, de la Banque européenne pour la reconstruction et le développement, du Royaumont Process, de la Black Sea Economic Co-operation, de la Central European Initiative, de la Southeast European Co-operation Initiative et du Southeast Europe Cooperation Process. 


\section{RÉSUMÉS}

The 1999 Southeast Europe Stability Pact aims at democratic and economic reforms and at the restructuring of existing agreements and relationships ; provisions concerning domestic security are examined. Objectives related to justice and domestic security include fighting corruption, managing immigration and asylum, and training police. Measures undertaken under the Southeast European Cooperation Initiative to facilitate regional cooperation against international crime are evaluated. These multilateral efforts have not succeeded. A bilateral approach shows more promise collaborative achievements between Italy and Albania illustrate both the potential and persisting challenges of bilateral strategies.

\section{INDEX}

Index géographique : Balkans, Europe centrale et orientale

Mots-clés : coopération internationale, Politique européenne de voisinage 